ON

\title{
THE ULCERATIVE PROCESS
}

\author{
IN \\ JOINTS. \\ BY C. ASTON KEY, \\ SURGEON TO GUY'S HOSPITAL, ETC. \\ READ MAY 14TH, 1833.
}

THE process of ulceration, like other inflammatory actions, is subject to certain laws, which have not escaped the acute observation of our great pathologist Mr. Hunter; though they cannot be said to have received that minute investigation to which the other terminations of inflammation have been submitted. The adhesive and suppurative forms of action have received from him the most careful examination; and the labours of subsequent pathologists tend to confirm the scientific view he has adopted, and so well explained in his work on the blood.

A principal advantage derived from the investigation of the pathological phenomena of inflammation, consists in observing the modification which they undergo according to the structure and texture of the parts engaged. Pathology is thus rendered a 
science of observation; and every honest and careful observer of phenomena, however humble and limited his observations may be, becomes a not unimportant contributor to the stores of medical science. With this feeling, I am induced to bring before the notice of the Society the observations I am about to offer on the process of ulceration, as it takes place in joints.

The ulcerative process is doubtless regulated by laws as fixed and certain as every other proceeding. in the animal economy; the difficulty of obtaining an accurate knowledge of them is perhaps greater, than the investigation of other inflammatory actions. In the adhesive inflammation, we have certain products which can be submitted to tests, and the progress of the action can be examined in all its stages and under every variety of attendant circumstance; but in a work of destruction, as the ulcerative pro. cess, the products of the action are removed from our observation; and the several steps by which the process is effected are with difficulty followed. Yet, we may observe, that the circumstances of structure, of texture, and the extent of vital organization with which parts are endowed, tend greatly to modify the relative disposition to ulcerative action.

The wisdom of this provision and the purposes of the animal economy to which it is subservient, are too apparent and too well known to the physiologist; to require being particularized. It is sufficient for voL. XVIII. 
my present object to point out three views in which ulceration may be regarded, or it might not be improperly said, three modes in which texture seems to modify the nature and course of this process: first, as it occurs in highly vascular structures; secondly, in parts possessing a somewhat lower degree of vascularity; and thirdly, as it is observed to take place in parts endowed with the least degree of organization.

The remarkable disposition to ulceration in those textures that are well supplied with blood, must be obvious, if not familiar, to us all. In the mucous membranes it is especially observable; these parts abound in vessels of large size, and are liable, under moderate degrees of inflammation, to pass into the ulcerative state; the mucous lining of the intestine, holding a first rank among vascular structures, quickly ulcerates under some forms of muco-enteritis; that of the trachea being somewhat less vascular, is less prone to ulceration.-The lining membrane of the mouth speedily exhibits an apthous surface, or even a deeper extent of ulceration, from trivial causes of inflammation; and the gums, disposed as they are to ulcerate, have this disposition still further increased, when they become spongy and more vascular.

There are some circumstances connected with this disposition to ulceration, that tend to throw some doubt on the received opinion, that the absorbents 
perform the office of removing parts that are under the influence of ulceration, and to refer some share, if not the whole, of this action to the veins. It is by no means satisfactorily ascertained what part the veins and the absorbents respectively take in healthy or nutritive absorption. In the absorption produced by disease, the nature of the process is still less definitely understood; and it yet remains a problem for the physiologist to solve, whether the veins are not mainly engaged, or at least assist, the absorbents, in the process of ulceration. The above examples favour this supposition; which is further strengthened by the fact, that all structures previously to being removed by ulceration, become unusually vascular; as if a more complete development of the sanguineous tissue were essential to this mode of absorption. We shall find, when describing articular ulceration, that this view of the process receives strong confirmation from the peculiar circumstances attending the ulceration of cartilage.

The action of the veins in producing ulceration of a villous surface, as that of the intestinal canal, is by no means rendered improbable by the membrane being abundantly furnished with absorbents. Gendrin* mentions that in those who have died with ulceration of the intestine, he has usually found the veins either filled with pus, or inflamed upon their inner surface. The same author relates an experi-

* Hist. Anat. des Inflammations, p. 707, tom 1. 
ment of injecting pus into the pleural cavity of an animal, and at the expiration of twenty-four hours finding, on dissection, a considerable quantity of the fluid in the branches of the thoracic veins. Other observations might be adduced in support of the opinion that the function of the absorbents is confined to nutrition, to the removal of interstitial fluids, and to the preservation of the form of the body during growth, or as Mr. Hunter has termed it, modelling absorption ; and that progressive absorption or ulceration is effected through the agency of the extreme branches of the venous system *.

* Sir Astley Cooper has in his collection an ulcer of the leg very successfully injected, in which the veins are developed in a remarkable manner ; they are numerous and large, and surround the margin of the ulcer. There is also in his collection another ulcer in which the absorbents of the leg have been injected; but they appear to be neither increased in size nor in number; the absorbents that in the sound state of the limb took their course through the site of the ulcer are cut through by the disease, and each absorbent can be seen to terminate in a vascular granulation.

Professor Coleman of the Veterinary College, at my request, made the following experiment. He caused to be inserted in the inner part of the thighs of an ass a rowel, which at the expiration of four days had established a copious suppuration. On the fourth day a small quantity of prussiate of potass was inserted in each sore and allowed to remain six hours, at the end of which period the animal was killed. To ascertain which of the two systems, the venous and the absorbent, had taken up most of the salt, I removed some blood from the iliac veins on both sides, and some from the mesenteric veins; and $\mathrm{Mr}$. Coleman's dissector collected half an ounce of fluid from the thoracic duct. These I submitted to Mr. Alfred Taylor, our 
In structures that present a less developed vascular tissue, the disposition to ulcerate under inflammation appears to be less determined than in the mucous textures. The serous membranes rarely afford evidence of ulceration on their exhaling surface. Pathologists who have written on the effects of inflammation in the different tissue, regard it as a somewhat rare occurrence; and when it does occur, as it occasionally does both on the pleura and peritoneum, it is the result of a complicated action, rather than the effect of a mere ulcerative process. The ulceration that is observed to commence on the peritoneal investment of the intestines, has its origin often in a scrofulous deposit, which leads to a degeneration of the membrane, and a total alteration of its structure, before the ulcerative process begins. A similar change in the texture of these membranes takes place, before they are removed by ulceration under the pressure of an abscess; adhesion of the

lecturer on medical jurisprudence and chemistry, who favoured me with the following analysis. "No. 1, the blood from the iliac veins, contains the prussiate in large proportion. No. 2, the serum from the thoracic duct contains it in about the ratio of $\frac{1}{5} \overline{0}$ of No. 1 ; and No. 3, the blood from the mesenteric veins, after standing six days, shews evidence of the prussiate in the ratio of about $\frac{1}{50}$ of No. 2, and therefore of about $\frac{1}{2500}$ of No. 1. The last specimen, No.3, did not at first exhibit any appearance indicative of the prussiate on the application of the reagent; but it is by no means unusual for the precipitate to shew itself after the lapse of a few days; the reaction being slow when the proportion of salt is minute, and any organic substance, as albumen, at the same time present." 
opposed serous surfaces is produced, and in examining the structures thus blended by the adhesive process, it is impossible to recognize the membranes; their organization has undergone a change, in order to fit them for the new process, in which they are about to be engaged.

The fibrous textures of the body, it is well known, exhibit a remarkable indisposition to ulceration; the length of time required for the separation of a dead portion of tendon, or for the pointing of a subfascial abscess, sufficiently evinces this. The long resistance which the intervertebral cartilage offers to the absorbent action caused by the pressure of an aneurismal sac, affords another example; the bones and the investing ligaments of the spinal column are frequently eroded to a considerable extent, while the fibro-cartilaginous structure often exhibits not any signs of incipient ulceration. In ligamentous fibre the process appears to be accompanied with some peculiar circumstances. The ligament, instead of preserving its usual form and size, becomes distended and feels pulpy. When cut into, the fibres are found to be separated from each other by a vascular structure, which upon being injected has a villous appearance. This interstitial vascular mass is the reticular membrane, that in the healthy structure unites the ligamentous fibres; under inflammation it becomes highly vascular, and assumes the appearance alluded to, while the fibres of the ligament retain their natural glistening appearance until, in the pro- 
gress of the disease, they at length become softened and pulpy previously to their undergoing absorption. It is not improbable that the ligamentous fibres themselves are passive in the ulcerative process, which there is some reason for believing is performed entirely by the vascular tissue that surrounds them.

The most striking illustration of this passive condition of parts that are undergoing the absorbing process, is afforded in the attempt made by nature to remove a cylindrical bone that has been rendered necrotic by inflammation. In making a section of a limb in this state, the first circumstance that arrests the attention is the smooth and polished condition of that portion of the dead bone that has been exposed to the atmosphere, while the other parts of its surface and its extremities present a number of holes or indentations, as if worm-eaten. When the sequestrum is removed from its case of new bone, and the interior of the latter is exposed to view, a number of flocculent bodies are seen attached to a membrane that supplies the newly formed bone. When injected these are shewn to be highly vascular, and are seen to fill the indentations in the dead bone. If the latter be carefully taken out of its case of new bone, these vascular elongations will be found to have a slight attachment to those parts of the dead bone in which they are imbedded.

It is by means of this organization that large cylindrical bones, when deprived of vitality, are found, 
in the course of time, to have undergone extensive absorption. The dead bone having no power of self-removal, the surrounding living parts are called upon to perform the office of removing the useless and offending mass; for this purpose the inner layer of the new structure which is to supply the place of the old, is furnished with an organization capable of effecting its removal; it becomes extremely vascular, resembling those structures, namely, the villous, which are known to possess, in a remarkable degree, the ulcerative disposition. The removal of dead bone, under these circumstances, has been attributed to a solvent power in the fluids effused; were this the cause of the gradual disappearance of the bone, its surface would be more uniformly dissolved, and would not present that worm-eaten appearance in those parts, to which the projections of the membrane are most closely applied.

In a manner analogous, in many respects, to the process of removing dead bone, does nature achieve the task of absorbing the cartilaginous structure, covering the articular extremities of bones. These structures possess but a low degree of organization; in their healthy condition they present very few of the characters of animal vitality ; they exhibit scarcely any trace of red blood-vessels, and for obvious reasons, their supply of nervous influence is not more than sufficient to connect them with the surrounding structures, as part of a whole. Under disease they exhibit that want of action which might be antici- 
pated from the limited extent of their organization. In acute inflammation of a joint, while the synovial membrane and ligaments are much altered, the cartilage appears unchanged in colour or in texture, and apparently uninfluenced by the increased action going on in the surrounding parts. The cartilage becomes, under disease, softer somewhat in texture; but this change may be as well attributed to the absence of pressure, as to the effect of inflammatory action; for healthy joints, when kept long at rest, are found to undergo a similar change, on their cartilaginous surface, from the want of that pressure to which they have been accustomed, and which may be necessary to the preservation of their due consistence. There are, however, some forms of inflammation under which the cartilage, very early in the disease, undergoes a change of structure; these instances are much less frequent, and may be looked upon as exceptions to the ordinary rule.

The peculiarity of organization is not the only circumstance that determines the disposition to the ulcerative process. There are other general causes that exert an influence in disposing parts to this form of action. Debility combined with inordinate action, or, as it is termed, irritability, which implies a disposition to action without proportionate power, is the morbid condition, on which this tendency in inflamed structure seems most to depend. Of this we see evidence, in the ulcerative disposition of newly formed parts, in the opening of recently healed ulcers, in 
the phagedenic tendency of ulcers in persons of excessively irritable constitution induced by irregularity of living, in the irritability attendant on most forms of caries of bone, and in many other diseases with which every surgeon is familiar. In scrofulous habits, this irritability is usually induced by disease, and disposes the parts affected with inflammation, to ulcerate.

These observations will tend to throw some light on the mode in which articular ulceration is effected; to which process $I$ now request the attention of the Society.

The progress of ulceration in cartilage covering the ends of bones, is not uniform in its course. The means, by which it is effected, vary according to the cause that gives rise to it. It is sometimes the result of acute synovial inflammation, or of a chronic affection of that membrane ; it is occasionally found as a primary affection, independent of the other textures of the joint; or it may be the result of disease commencing in the subjacent bone, and extending to the under surface of the cartilage. All these circumstances tend to modify the course which nature adopts in the removal of cartilage by ulceration. There are four modes in which it takes place, these are sometimes found to occur, more or less, together in the same joint. I shall first describe the most common form; that, in which it occurs as a consequence of inflammation of the synovial membrane.

I am inclined to believe that inflammation of this 
membrane is the most frequent cause of ulceration of the cartilage. This opinion I have been led to adopt from the examination of a considerable number of diseased joints, in which ulceration of cartilage has been found to exist in different degrees of progress, from its most advanced stage, in which the bone has been entirely denuded, to the very incipient abrasion of its surface or margin. The history of some of these cases, together with the morbid appearances, has also satisfactorily proved the existence of a long continued synovial affection, before any alteration of the cartilaginous surface could have taken place; as the cartilage in some has been quite sound, with the exception of a slight loss of substance at the edge of the bone, where the synovial membrane is reflected from it; while the symptoms of diseased joint have existed for many months, with pain over a large part of the synovial surface, and general swelling of the joint. It is not uncommon to find the extremity of one bone extensively ulcerated, especially in the knee joint, while the other may exhibit the same disease in an incipient state, and thus shew the course which the diseased action has taken. The tibia is not unfrequently seen wholly deprived of its cartilage, and one or both semilunar cartilages destroyed, while the cartilage of the femur is but partially denuded, and that of the patella sound, with the exception of its margin, which has evidently suffered in texture from its continuity with the altered synovial membrane.

The inflammation of the synovial membrane that leads to ulceration of cartilage in the ordinary 
strumous affection of joints in the adult, is not, as far as my observation goes, of the most acute kind. This form of inflammation in most cases is readily controlled by remedies ; probably, because it is more early attended to than subacute inflammation, and treatment more promptly applied. The less acute forms of the disease, assuming various shades of activity between the chronic and the acute forms, rarely occur for any great length of time without the cartilage participating in the mischief. This may in some measure depend on the peculiarity of those constitutions, in which subacute inflammation seems to have a spontaneous origin.

The knee joint is most frequently observed to suffer disorganization from this form of inflammation. When taken in the early stage, the subacute inflammation of the membrane readily yields to a judicious administration of mercurial remedies combined with moderate depletion and remedies that allay that excessive irritability of the system, that generally attends inflammation of the synovial membrane in delicate persons. When the more acute symptoms are subdued, the membrane sometimes fails to regain its normal condition, passing into a chronic form of action so slight, as to attract but little attention, and often regarded as stiffness that will yield to exercise and passive motion. This slight degree of inflammation that remains often lays the foundation of future mischief, especially if the condition of the patient's health is not adverted to, after the acute stage of the inflammation has subsided. 
The nature of the remedies employed always leaves the patient in a state of weakness and irritability, under which the low degree of action that remains in the joint will be disposed to assume the ulcerative form. It is in this state that the compound preparations of sarsaparilla with alkalis are of so much benefit; there is no medicine that more effectually removes this irritable state of system by giving vigour and allaying excessive action. This state of joint, as the disease advances, is usually attended with more pain, than when the disease assumes from the commencement the chronic form; the intervals of ease become short and few ; and the action goes on with but little interruption to the formation of abscess.

In the chronic form of synovial inflammation that occurs in indolent habits of a strumous tendency, especially persons below the age of puberty, years often elapse before the ulcerative process is completed. The symptoms are proportionally mild in their course: The joint is not much swelled; the general and uniform fullness of the joint, so characteristic, as $\mathbf{M r}$. Brodie has observed, of the most acute forms of inflammation of the synovial membrane, is absent; the joint appears as if the bones themselves were enlarged, an appearance as much produced by the shrinking of the limb above and below the joint, as by the swelling of the joint itself. The swelling of the soft parts about the joint depends on the degree of inflammation present in the synovial membrane and the consequent effusion in the soft parts. In the 
most chronic forms the bones can almost be felt through their ligamentous investments; in the less chronic forms, when the disease runs its course in a shorter period, there is effusion of albumen in the soft structures surrounding the joint, which increases its volume, preventing the bones being distinctly felt, and in some measure altering the form of the joint.

It would appear then that there are three forms of inflammation (hitherto considered) of the synovial membrane leading to ulceration of the cartilage: the acute form becoming chronic, in which the joint retains the appearance of uniform swelling characteristic of affections of the synovial membrane; secondly, the chronic form in which the disease is insidious and slow in its progress, and the swelling of the soft parts inconsiderable; and thirdly, the subacute form, intermediate between the two former, marked by more activity, attended with more swelling and more pain than the very chronic form, and a more rapid disorganization of the structure of the joint.

The first circumstance that strikes us on opening a diseased joint is, the different degrees of ulceration in the articular surfaces, and the different extent to which the interarticular cartilage and ligaments have suffered. This will depend upon the part in which the diseased action has commenced, which perhaps in most cases is determined by accident, as the seat of the blow or sprain which may have excited the in- 
flammation, or the form of the joint producing unequal bearing upon the surfaces, and thus determining the inflammation to that part where the pressure is greatest. The inner part of the knee joint usually exhibits the most extensive ulceration on account of the oblique bearing of the femur, and its consequently unequal pressure on the inner part of the head of the tibia. We therefore find the inner semilunar cartilage more often destroyed than the outer, and a corresponding destruction of the cartilage covering the inner condyle of the femur and inner part of the head of the tibia. The patella and the extremity of the femur are the parts on which the ulcerative process can be best traced on account of the disease being in these less advanced. In the former bone the first part that commonly gives way to ulceration is the margin of the cartilage, where the synovial membrane is reflected from it. At this point sulci of different depths are formed which cannot be always distinguished, until the thickened edge of the synovial membrane is raised. The ulcerated surface sometimes exhibit parallel vascular lines verging towards the centre, and having their origin from the synovial membrane. The synovial membrane at this part, if the vessels are well filled with fine injection, appears highly vascular, and fringed or villous like a mucous membrane. This increased vascularity is particularly noticeable at the edge of the membrane, and in these portions of the fringed margin that correspond to the ulcerated surface of the cartilage ; the other parts of the synovial membrane have their 
vascularity but slightly increased. This highly vascular fringe of membrane is a newly organized, and will be found in some parts to be a superadded, structure, for the purpose of producing ulceration of the contiguous cartilage. It may when recently formed be raised in some parts from the synovial membrane, but is found to adhere very slightly to that part of the cartilage where ulceration is going on; this adhesion is not perceived, unless the joint is opened with care. The nature of the process will be best understood by a reference to the patella*.

The lower part of the bone exhibits extensive traces of ulceration of its cartilage. Nearly the whole of its margin also shews signs of ulceration having commenced, with the exception of a small portion at the upper part, where the synovial membrane appears to have been converted into a substance like cartilage (b). The parts marked $c, d$, and $e$, are deeply furrowed, and the synovial membrane at these parts is fully developed and organized in the manner above described. The margin of the membrane at $a$ is highly vascular, and was found in the form of two or three fringed projections filling up the space where ulceration was proceeding. In the same manner the surface of the membrane at $c$, which is drawn down, was seen to overlap and partly to adhere to the cartilage at $d$. At $e$ the same adaptation of the synovial membrane was observable, and the preparation from which the drawing was made still shews

* See Plate II. 
these points. At $d$ the surface of the cartilage appears vascular; this arises from the production of a new membrane continuous with the synovial membrane and formed from it. Its attachment to the latter has been destroyed; but its connexion with and its supply of vessels from the synovial membrane at $b$ was distinctly seen when the joint was opened. This new production of the synovial membrane performs the same office as the vascular edge of the latter, namely, the absorption of the surface of the cartilage. This membrane may be readily seen either partially developed or completely formed on the cartilaginous surface in the examination of diseased joints. It is frequently seen covering one or both condyles of the femur.

The process therefore by which the ulceration of cartilage is in this case effected, is analogous to that by which the sequestrum of the cylindrical bones under necrosis takes place. Indisposed to ulceration from the low degree of its organization, it is acted upon by the newly organized synovial surface, which is rendered highly vascular, and by means of its villous processes forms a groove in the edge of the cartilage, thus commencing the work of destruction. The cartilage at the edge is sometimes entirely destroyed so as to lay bare the bone; in which case vascular granulations also arise from the surface of the exposed bone and assist the membrane in the work of absorption. This, however, is more usually to be observed in the most acute form of in- 
flammation. In the more chronic form the vascular fringe of synovial membrane contracts adhesion to the surface of the cartilage in which ulceration is going on, and gives rise to the formation of a new membrane, which spreads gradually over the surface of the cartilage. A diseased joint is hardly ever examined without exhibiting one or more of the bones partly covered with this pulpy membrane. When injected, its vascularity is found to vary according to the activity of the inflammation in the joint; when first formed it exhibits considerable vascularity, during the ulcerative process; when the cartilage has been wholly absorbed, and the ulcerative process has been checked by the inflammation being arrested, this membrane then serves another purpose; it becomes the medium of union between opposed surfaces of bone, or the means of anchylosis. Long after all inflammation has subsided, one of the condyles of the femur is often found adhering to the tibia by means of this membrane, which appears white and ligamentous, a layer of cartilage often remaining between the membrane and the bones, as if the process of ulceration were arrested.

A membrane is sometimes seen in joints under different circumstances, and affords a contrast to the above membrane, as well in structure as in its office. I allude to that adventitious membrane that is formed from the edge of the synovial membrane, in consequence of inflammation of a joint, induced by a contiguous disease of bone, as necrosis. In this case 
the membrane is formed for the purpose of circumscribing the cavity of the joint, when the cartilage is destroyed by the extension of the disease. It possesses but little vascularity, is smooth on its surface, not being furnished with the villous texture necessary to the ulcerative function. The opposed cartilage under these circumstances appears entire, ulceration taking place only on the surface next to the bone, and the membrane has not any connexion with the surface of the cartilage.

The formation of the vascular membrane frequently takes place without suppuration; as may be seen in strumous joints that have been the subject of chronic inflammation for years, without abscess having formed; and the inflammation is sometimes confined to one side of the joint. Such joints are sometimes seized with an acute attack of inflammation of that part which had been previously healthy; suppuration rapidly ensues, under which the failing of the patient's health and powers demand amputation of the limb for the preservation of life. The two sides of the joint present different appearances; one shews no signs of recent inflammation; the ends of the bones, are partially, perhaps wholly, deprived of their cartilage, or the cartilaginous surface is ulcerated only to a certain depth; between the bones is seen the membrane adhering to the cartilage, white, possessing scarcely a trace of vascularity, and merely serving to connect the ends of the bones by means of what is termed ligamentous anchylosis. The other side of 
the joint is full of pus; every tissue in a state of active inflammation; the cartilage removed by a rapid process of ulceration, in which the bone is probably found to have taken an active part; and the ends of the bone are seen covered with vascular fungous granulations, from which pus is abundantly secreted.

This secondary attack of inflammation in the kneejoint, is often caused by neglecting the very important precaution of supporting the weak joint, after it has recovered from the long previous inflammation. Long continued inflammation, of which we see such frequent examples, rarely fails to be attended, after a lapse of years, with partial ligamentous anchylosis of that part of the joint which has been the seat of the affection. From careful observation of a large number of these cases, and subsequent examination of the joints, I believe that the ulcerative and anchylosing process may be going on for many years. Nature seems to have in view, as her ultimate object, the complete anchylosis of the surface of the joint; this she effects with the least possible degree of inflammation, and her task is often completed with little disturbance of the patient's general health, except occasionally slight attacks of pain and some swelling about the joint, that subside under rest and mild treatment. But it is necessary in order to bring the process to a successful termination, to favour the process by restraining the limb from motion, and by giving due support to the joint to prevent any accidental strain or extension of the interior struc- 
tures of the joint, which may occasion acute inflammation. This can only be effected by means of a firm splint of wood or metal, combined with straps of plaster and mercurial ointment applied in the manner recommended by Mr. Scott. The latter applications, however, are more successful in reducing chronic inflammation in its earlier stage, before the membrane is formed; when this has taken place, support with pressure, and a small issue, if it can be borne, are more advantageous. With this security given to the joint, the patient is enabled to take moderate exercise, inflammatory attacks are in a great measure prevented, and he may confidently look forward to the possession of a very useful though partly stiff limb.

The destruction of the semilunar cartilages in its process resembles that of the intervertebral substance of the spine and other similar fibro cartilaginous structures. It is preceded by inflamrnation of the contiguous synovial membrane, which is followed by softening of the cartilage. It seems to be rather a process of slow degeneration than of progressive ulceration. They are sometimes destroyed very early in the disease; the first symptom of the affection of the joint is pain about the head of the tibia, and on each side of the ligamentum patellæ, with effusion about this part of the joint. In acute inflammation, on the contrary, the cartilages are not unfrequently found entire, while those covering the surfaces of the bones have undergone extensive ulceration. 
The hip-joint is less frequently the subject of acute than of chronic inflammation, probably, from being well protected from the influence of atmospheric changes, to which the knee and most other joints are exposed. In the adult, acute disease of this joint is occasionally seen, in which the whole structure of the joint, cartilages as well as ligament, undergoes complete disorganization in the space of a few weeks. But the hip disease, emphatically so called, is a chronic affection uniformly attended with ulceration of the cartilage.

Mr. Brodie, who has given a most clear and correct account of the symptoms and progress of the disease, regards it as a primary affection of the cartilage. The opportunities which present themselves to any individual, of observing this disease in its early stages by dissection, must necessarily be few. The cases, which it has fallen to my lot to examine, have induced me to believe that the ulceration of the cartilage is preceded by inflammation of the ligamentum teres. In the drawing of the early stage of this disease, which I lay before the Society, will be seen the usual morbid appearances that I have met with. This joint was taken from a young female who for six months prior to her death had laboured under the usual symptoms of chronic inflammation of the hipjoint. The symptoms had partly yielded to the treatment employed, when she was attacked with another disease of which she died. The ligamentum teres was found much thicker and more pulpy than 
usual, from interstitial effusion, the vessels upon its investing synovial membrane were distinct and large, without being filled with injection. At the root of the ligament, where it is attached to the head of the femur, a spot of ulceration in the cartilage is seen, commencing, as it does in other joints, by an extension of the vessels in form of a membrane from the root of the vascular ligament. The same process was also taking place on the acetabulum, where the ligamentum teres is attached.

I cannot undertake to say, that the hip disease shall, in every instance, present these morbid appearances, or that cases do not occur in which ulceration exists as a primary disease, without any affection of the ligament or synovial membrane. Mr. Brodie's assertion that it does exist as a primary disease, coming from so excellent a pathologist, is sufficient to substantiate the fact. But observation of this disease in its different stages, and of the mode in which the disease is brought into action, together with the post mortem appearances, affords strong proof, that, at least in many instances, the action is propagated from the ligament to the cartilage, and that ulceration of the latter is consequent upon inflammation of the former.

The beginning of the affection is frequently to be traced to a fall, by which the legs have been forcibly separated, and the ligamentum teres stretched. In some cases, the injury has been so considerable, as to 
occasion the patient to rest the limb for some days, on account of the severity of the pain. This to a certain extent subsides; and the inflammation that remains, assumes the chronic form. If the patient's health is good, he recovers with only a slight temporary weakness in the joint; in the more feeble habit with a tendency to strenuous action, the disease gradually passes into the ulcerative form. Sometimes from the tender age of the child, no cause can be assigned for the disease ; perhaps, in some instances, it may have a purely constitutional origin. The motions of the joint, that give the patient most pain, are strongly indicative of the seat of the affection; in the earliest stage, before the soft parts could well be affected, if the disease commenced in the cartilage, eversion of the thigh, and abduction of the limb from the other, produce the greatest degree of suffering to the patient; while he can bear the joint to be flexed, and to be slightly inverted, without complaining. A similar indication of the ligamentum teres being inflamed, is the pain sometimes expressed on pressing the head of the femur against the acetabulum; in its healthy state the ligament, being lodged in the hollow of the acetabulum, receives but little pressure; but when it is swelled by inflammation, the cavity of the joint affords it less protection, and when pressure is made by forcing the head of the femur upwards, the ligament is compressed, and usually produces some degree of pain. The circumstance, too, of the ligamentum teres being destroyed by ulceration, when the head of the bone and acetabulum are only 
partially ulcerated, may be considered as presumptive proof of it being very early engaged in the disease. There are few cases of post mortem inspection of the hip joint in an advanced stage of disease, in which the ligamentum teres is not found destroyed.

There is a disease about the tarsus, the commencement and progress of which resembles, in many points, that of the hip joint. It is usually considered as a disease of the ancle joint itself ; but it has its origin in the articulation of the under surface of the astragalus with the os calcis. The inflammation is slow in its progress, and when fairly established in the joint of a strumous subject, rarely fails to end in destruction of its cartilage, and of the interarticular ligament connecting these bones together. It is usually attributed to a sprain, or twist of the foot sidewise, as if the ligaments had been injured; this joint has extensive lateral motion, and is restrained in its movements by this ligament. Very early in the disease, the least stretch of the ligament causes acute pain, and examination of the joint, where the opportunity offers, also proves it to be inflamed and thickened and often extensively disorganized. The mischief is generally not confined to this joint, but extends to the upper surface of the astragalus. The ancle joint however, in the cases which $I$ have had an opportunity of examining, has suffered much less from ulceration of its cartilage, than the lower joint; and I believe that in many of the cases, which are regarded as disease of the ancle, the action will be 
found to have its origin in the interarticular ligaments connecting the os calcis and astragalus, and extending, as in the hip joint, from it to the surface of the cartilage. The vascular membrane, which the synovial investment of the ligament assists in forming, may also be distinctly traced.

The second mode in which nature effects the ulceration of cartilage without the agency of its own vessels, may be seen in the rapid process of disorganization that follows a wound into a joint.

Under chronic strumous action there is time allowed for the gradual developement of a membrane that extends itself over the cartilage, and in its progress absorbs it. But in acute inflammation followed by suppuration the process is modified, and affords, perhaps, the best and clearest illustration of that mode of ulceration which it is the object of this paper to explain.

When suppuration follows acute inflammation from a wound of the synovial membrane, the latter undergoes that change which enables it to perform its new function. The surface becomes highly vascular, and in most parts covered with a new deposit of adhesive matter, which adheres firmly to the synovial membrane. The new surface is irregular, wanting the polish of the original membrane, and appears in many parts villous or furnished with vascular fringed projections. In a joint thus far advanced in disease, the 
only mode of arresting the disease, or of repairing the mischief occasioned by the inflammation, consists in the production of anchylosis. To this end the removal of the cartilage is an essential step; and it would appear that the office of removing it devolves on the inflamed synovial membrane. The cartilage, under these circumstances, is not only eroded at the edge where the synovial membrane is reflected from it; but grooves and indentations may be traced in various parts of it, having no connection, as in the progressive strumous form of ulceration, with the edge of the synovial membrane, and not shewing any indications of a new membrane forming on its surface.

The means by which this ulceration is effected appear to be the newly organized surface of the synovial membrane in contact with the parts in which absorption is going on. To those who will carefully examine joints in this condition, the evidence of this will, I think, be sufficiently conclusive. The absence of all action in the cartilage and a total want of vascularity in those parts where ulceration appears to be most active, were the circumstances that first led me to look for some agent in the work of ulceration. The ulceration evidently begins on the surface of the cartilage, and not on that side next to the bone. It presents merely an eroded surface; there is no disorganization of its texture in the parts where absorption is about to take place; there is no previous degeneration of the cartilage into its primary fibrous structure, as may be seen in other forms of ulcera- 
tion; but the cartilage seems to have lost part of its surface, as if it had been dug out; the remaining part appearing healthy, and presenting no trace of increased vascularity. The grooves are found only in those parts of the cartilage that happen to be opposed to the fringed and vascular synovial membrane; and these highly organized portions of the membrane may be seen to be closely adapted, and even to fit into the grooves in the cartilage. Those parts of the cartilage that happen to be in contact with another cartilaginous surface present no signs or trace of ulceration, but appear to the eye perfectly healthy, and in texture firm. The process exhibits the closest analogy to that by which nature removes dead bones; the same inactivity or passive condition of the parts absorbed; the same suppurative action from the vascular granulation; and a similar degree of vascularity bestowed upon the newly organized structure, which has to perform the office of absorption.

It is not, however, in every instance of suppuration in a joint, even where this villous membrane is found, that ulceration of the opposite cartilage is to be looked for as an uniform occurrence, for strumous joints are occasionally examined, in which the synovial capsule has been for many months distended with purulent secretion, and the synovial membrane covered with flocculi hanging into the joint, without a trace of ulceration in the opposite cartilaginous surface. This exception rather favours the view which $I$ have advanced of the ulcerative process in the case of suppu- 
ration from wound. The condition essential to the act of absorption is here wanting. There is not a wound or opening by which the pus can escape as fast as it is secreted; it consequently collects in the cavity of the joint, and, by distension, prevents the membrane coming in contact with the cartilage; and the villous projections from the membrane, even when the vessels are well filled with fine injection, do not exhibit that degree of vascularity which is so clearly developed when ulceration of the cartilage takes place. This little comparative vascularity accords with the suppurative action being slow and proceeding without pain or the other signs of inflammation.

The knee joint, which first led me to suspect the action of the membrane in this form of ulceration, and of which I have given a drawing, was removed by amputation between four and five years ago on account of an accident. The patient, a healthy man, about 34 years of age, was thrown with force from the seat of a cabriolet which he was driving, and cut the outer part of the knee by coming in contact with a sharp stone. He came to the surgery of the hospital, and having had the wound dressed, returned to his occupation. In the course of a few days, finding that his knee had swelled considerably, and had become very painful, he came to the hospital for admission, and was placed under my care. The capsule of the joint was distended with fluid, and exquisitely sensitive on pressure being 
made; the wound in the integuments had partly healed, but looked unhealthy; his pulse 120 in a minute; his tongue, skin, and countenance bearing every indication of fever. The suspicion, which I entertained of the synovial membrane being wounded was soon confirmed by a rapid process of ulceration in the membrane, and an extensive burrowing of pus along the back part of the leg under the gastrocnemii muscles, and also under the extensor muscles on the fore part of the femur. His condition being such as to preclude all chance of saving his life without amputation, at the end of the third week the operation was performed, and with ultimate success. The joint, which had been carefully injected with fine injection soon after the operation, was examined on the following day. On being laid open, it appeared to contain scarcely any pus, the surfaces of the parts forming the articulation being in close contact. On turning the patella down by making two lateral incisions, the singular appearance of the inner condyle of the femur forcibly struck those who were present. Deep grooves of ulceration had been formed on its surface as if, to use Mr. Brodie's expression, portions of the cartilage had been chiselled out; and although the injection had filled the smallest vessels of the inflamed synovial membrane, not a trace of vascularity could be distinguished either upon or in the neighbourhood of the ulcerated grooves. The outer condyle exhibited the same appearances, but in a less degree. 
On replacing the patella, it appeared that only those parts of the cartilage had suffered a loss of substance, that were opposed to the inflamed synovial membrane ; the surface of the patella and the corresponding trochlea of the femur retaining their integrity and natural appearance. The synovial membrane opposite to the grooves in the femur was covered with an irregular layer of membrane presenting several fringed projections, that fitted into the grooves of the absorbed cartilage, and at the points where ulceration had been most active, the flocculi of the membrane appeared to be most vascular. At the points $f$ and $g$ will be seen a corresponding portion of cartilage and membrane, the former free from ulceration, and the latter smooth, and but little vascular; while the contiguous surface of the cartilage at $b$ and $c$, and the membrane at $a$ and $d$ have undergone a change, that cannot but be regarded in the relation of cause and effect. A process of ulceration has also commenced at the margin of the cartilage at $h$, where the synovial membrane shews unusual vascularity, and similar appearances are observable at the whole circumference of the patella. In carefully looking over this joint, I could not discover any part of the cartilage in a state of ulceration, to which the synovial membrane had not access; and wherever the fringed vascular membrane had applied itself closely to the opposite cartilage, ulceration appeared to have made more or less progress.

Nature, it seems, does not often adopt this mode of 
removing the cartilage. It is only in the acute form of inflammation, as in wounds of joints, that $I$ have observed it. The process of removing the cartilage appears at all times and under all circumstances of disease, an object that she endeavours to accomplish; while the cartilage remains entire, anchylosis, the natural cure in some forms of diseased joint, cannot be effected; and therefore we may often observe ulceration of cartilage going on very early in those diseases, that from some defect in the patient's constitution cannot be arrested without anchylosis. The means by which nature effects her purpose of removing the cartilage, which being unable to take an active share in the process of anchylosis is an impediment in the way of her process, will be found to vary according to the activity of the inflammation and the consequent rapidity of the process. In the most chronic form of strumous ulceration, the removal of the cartilage is effected by the gradual developement and organization of the synovial membrane where it is reflected from the edge of the cartilage, as I have endeavoured to explain in a former part of this paper. Where the process is required to be more rapid, a false membrane is effused from the edge of the synovial membrane, that gradually diffuses itself over the whole surface of the cartilage, and by means of its increased vascularity, ulcerates the cartilage even to the bone, anastomosing often with the granulations of the exposed cancellated structure. In the most intense forms of inflammation, all her resources are called to her assistance; 
the ulcerative process is not confined to the margin of the cartilage or to the action of a membrane having its origin from the margin; but, the opposite synovial surface is furnished with the necessary organization, and takes an active share in the work of destruction.

'The degeneration of the cartilage of a joint into a fibrous structure is, as far as my observations have enabled me to judge, a disease of a peculiar character, and differing in many respects from the ordinary affections of joints that end in the destruction of the cartilage. I have had but few opportunities of verifying by dissection, the existence of this disease. Mr. Brodie has described it, and appears to regard it as a not uncommon occurrence; in one instance he found it combined with disease of the intervertebral substance. Of three cases that have come under my notice, two occurred in subjects brought into the dissecting room, and the history of which I was uracquainted with; the other case was that of a gentleman who was labouring under stone in the bladder, and suddenly experienced a severe attack of pain about the head of the fibula and the bursæ at the back part of the head of the tibia. The pain was accompanied with considerable fever, and slight swelling of the parts in which he complained of the pain. On the third day the pain shifted from the fibula to the knee joint, which swelled as if from an effusion of synovial vol. XvirI. 
fluid. The suffering now became excessive, and the fever assumed the typhoid character. At the end of ten days from the commencement of the attack, he died. The bladder presented less evidence of inflammatory action than might have been expected from the intensity of his sufferings. The knee joint was distended with a thin opaque synovial secretion of a somewhat puriform character; the surface of the synovial membrane presented here and there patches of more than ordinary vascularity. The cartilaginous surfaces of the bones were entire with the exception of a small spot on the end of the femur, which appeared ragged, and irregularly broken up into a fibrous mass.

This change in the cartilage is analogous to that which the intervertebral substance undergoes under scrofulous action. I have not had opportunities of examining the condition of the synovial membrane in this affection.

The removal of the cartilage from the heads of bones in old people, proceeds so slowly that it is difficult to say, on the examination of a joint, whether the action has ceased, or is still in a state of progress. The form of disease to which I allude, is attended with a good deal of stiffening of the joint, accompanied by what are termed rheumatic pains. The place of the cartilage is often supplied by a bony deposit resembling ivory in texture as well as appearance. I have in one case seen the synovial membrane 
at its margin fringed and vascular, and apparently engaged in the ulcerative process. It was in a shoulder joint of a gentleman nearly sixty years of age, who had met with an accident to the shoulder, by which the actions of the joint had been impaired in consequence of subsequent chronic inflammation. I am unable to offer any very conclusive evidence as to the exact nature of the process in all such cases; but $I$ believe it will be found to be analogous to the processes which I have previously described.

The last form, in which ulceration of cartilage takes place, is that which commences on the surface of the cartilage attached to the bone.

"In this disease of the joints," says Mr. Brodie, " the cancellous structure of the bones is the part primarily affected; in consequence of which ulceration takes place in the cartilage covering their articulating surfaces. The cartilage being ulcerated, the subsequent progress of the disease is in many respects the same, as where this ulceration takes place in the first instance." *

There are two forms of disease in the bone under which this secondary absorption of cartilage takes place; the one is of a chronic nature; the other

* Brodie on Diseased Joints, p. 210. 
assumes an acute form ; but in the process of ulceration the same passive condition of the cartilage may be observed, as in that which commences within the cavity of the joint.

- The chronic form is that in which a strumous action takes place in the cancellated structure, " which becomes preternaturally vascular, and contains a less than usual quantity of earthy matter; at first a transparent fluid, and afterwards a yellow cheesy substance is deposited in the cancelli." * When the cartilage begins to give way, vessels can be seen shooting towards it, and accumulate in sufficient number to form a vascular tissue covering the attached surface of the cartilage. I have never examined a joint, in which disease appeared to have begun in the cancelli, and in which ulceration commenced on the surface of the cartilage within the joint. The ulcerative process having opened, or nearly so, the surface of the cartilage towards the joint, the synovial membrane becomes inflamed, and the ulceration is then forwarded by a similar process commencing at the edge of the cartilage, by means of the synovial membrane and a newly developed vascular structure, as has been previously described.

The bones, in which I have observed this form of disease, are the small bones of the carpus and the extremity of the femur; and more frequently, the 
head of the tibia, and the bones of the tarsus. Persons of all ages appear to be liable to it ; I have witnessed it in patients from the age of two years to fifty. The subjects of it are those who appear to possess in a marked degree the scrofulous diathesis. The symptoms of the disease and its progress are, unquestionably, familiar to the surgeon, from the accurate and clear description of Mr. Brodie.

It is unnecessary to make any further observations on this form of ulceration, as I apprehend that most pathologists will concur in the probability of the loss of the cartilage being effected by means of the vascular granulations that spring up from the cancelli, and appear to form a continuous structure with the surface of the cartilage. In making a transverse section of a joint under these circumstances, there is no trace to be seen of increased vascularity in the cartilage, nor in the synovial membrane until the action is far advanced, that could lead us to suppose, that the cartilage was ulcerated by any other agent than the vascular tissue of the bone.

The acute form of the disease differs from the former in the comparative suddenness of the attack, as well as in the appearance which the bone presents. The former is like all strumous affections, slow in its progress, and at first marked by little or no pain in the part. Months often elapse, before the symptoms become severe, and the constitution much affected. But in the acute form of disease attacking the spongy 
extremities of bones, the pain is often severe in the beginning, the limb at that part tender when pressed, and the constitution a good deal disturbed. A few weeks only elapse before the joint exhibits symptoms of participating in the mischief. From this time the disease makes rapid progress; if suppuration takes place in the cavity of the joint, the synovial membrane ulcerates, and allows the matter to burrow between the muscles of the limb; fistulous openings at length form, and tend in some measure to abate the patient's sufferings. The effect, however, upon the constitution is such, that amputation is usually resorted to for the preservation of life.

Examination of such a joint exhibits very different appearances from those which are observed in the chronic strumous disease of the bone; not in the cavity of the joint itself, for here the process of destruction is in some respects the same; but in the bone the affection is found to be altogether of a different character. The substance of the bone retains its firmness of texture; and when cut through shews no signs of disease except at one part of the cancelli. There a cavity is found containing one or more portions of detached bone, surrounded with pus; this cavity is found to communicate with the joint by a fistulous opening of small size, which may sometimes escape observation. The cancellated structure of the bone surrounding the cavity usually appears natural and sending forth vascular granulations. The cartilage covering the end of the bone is extensively ulcerated 
in some parts, while in others it appears to have undergone no change. The process of ulceration evidently begins on the outside of the joint, for the cartilage when closely examined, appears to be undermined, and the surface towards the joint where this undermining process is going on, seems quite sound. The synovial membrane shews signs of acute inflammation, and its cavity is found to communicate with one or more extensive collections of pus above and below the joint.

This form of disease is in its nature analogous to necrosis of the shafts of the cylindrical bones. The cancellated structure of the spongy ends of these bones, is not unfrequently the seat of inflammation, from accident or from idiopathic causes, which ends in the death of a small portion of the structure. If the disease commence above the epiphysis, an abscess forms externally, and the dead portion escapes at the aperture, in the form of a spongy mass consisting of little else than earthy matter. The opening rapidly heals after the exciting cause is removed. The progress of the case, however, is very different when the epiphysis itself is inflamed, and death ensues in some portion of its spongy texture. The abscess which forms around the dead mass, finds difficulty in forming an outlet externally through the dense ligamentous structure that invests the sides of the epiphysis, and thus the cavity of the joint becomes endangered by the pressure of the matter towards the cartilaginous surface. The bone undergoes gradual absorp- 
tion until the pus reaches the cartilage; then for a time the process becomes checked, but as granulations spring up from the surrounding cancelli, the cartilage yields to their influence, and the cavity of the joint at length communicates with the purulent depôt in the substance of the bone.

It is not improbable, from what is observed in the process of necrosis in the shafts of long bones, that the dead portion may be absorbed by the surrounding living parts, and thus prevent the necessity of the abscess bursting into the cavity of the joint. Our means of forwarding this desirable termination of the disease, in some joints, are limited to such as diminish inflammatory action; while in others, as in the head of the tibia and in the trochanter, the trephine may be employed with the best possible effect; and if applied early in the disease, may prevent the disorganization of the joint.

The drawing represents the appearance and condition of a bone under this state of disease. The bone is that of a child, whose femur had been attacked with inflammation, which rapidly spread to the knee joint. From the imperfect history of the case afforded by the parents, it appeared that it had been considered from the commencement as a case of articular inflammation; and in so young a subject, whose bones are so well covered with adeps, it may be at first difficult to distinguish accurately between a disease of the epiphysis and of the synovial mem- 
brane. When admitted into the hospital, it presented the usual appearances of advanced articular suppuration; two considerable fistulous openings were formed to communicate with the joint, and also with a large abscess that extended up the thigh. The child's health precluding all chance of recovery, except from a speedy removal of the disease, amputation was performed. The cavity in the bone and the dead portion of the cancellated structure which were discovered by a perpendicular section of the condyle, sufficiently explains the nature and cause of the diseased action of the joint. The appearance of the cartilage I would particularly bring before the notice of the Society. It appears wholly free from vascularity, though the part has been injected with size and vermilion; and the surface seems healthy; the parts where ulceration is going on, appear undermined, and the spots of ulceration are filled up with a tuft of vascular granulations, which are in all probability the agents by which the cartilage has been removed. The appearances are strikingly analogous to those observed in the section of a dead cylindrical bone which is undergoing absorption. 